\title{
EXAMPLES OF SUBFACTORS WITH PROPERTY T STANDARD INVARIANT
}

\author{
DIETMAR BISCH${ }^{1}$ AND SORIN POPA ${ }^{2}$ \\ UC Santa Barbara \\ Department of Mathematics \\ Santa Barbara, CA 93106
}

\author{
Université de Genève \\ UCLA \\ Section de mathématiques \\ and \\ Department of Mathematics \\ CH-1211 Genève 24 \\ Los Angeles, CA 90024
}

\begin{abstract}
Let $H$ and $K$ be two finite groups with a properly outer action on the $\mathrm{II}_{1}$ factor $M$. We prove that the group type inclusions $M^{H} \subset M \rtimes K$, studied in detail in $[\mathrm{BH}]$, have property $T$ in the sense of [Po6] if and only if the group generated by $H$ and $K$ in the outer automorphism group of $M$ has Kazhdan's property T [K]. This construction yields irreducible, infinite depth subfactors with small Jones indices and property $\mathrm{T}$ standard invariant.
\end{abstract}

\section{INTRODUCTION}

If $H$ and $K$ are two finite groups with a properly outer action on the $\mathrm{II}_{1}$ factor $M$, we can compose the two subfactors $M^{H} \subset M$ and $M \subset M \rtimes K$ to obtain a new inclusion $M^{H} \subset M \rtimes K$. While the Jones index of $M^{H} \subset M \rtimes K$ is finite, being equal to $|H| \cdot|K|$, in general this inclusion can no longer be obtained as a fixed point algebra (or a crossed product) by a group action, or more generally a coaction of a Kac algebra. It was shown in $[\mathrm{BH}]$ that several interesting properties of these group type inclusions are determined by the group $G$ generated by $H$ and $K$ in the outer automorphism group of the factor $M$. For instance, it was shown there that $M^{H} \subset M \rtimes K$ is amenable (resp. has finite depth) if and only if $G$ is an amenable (resp. finite) group. Since any second countable discrete group has a properly outer action on the hyperfinite $\mathrm{II}_{1}$ factor $R$, numerous examples of subfactors, whose standard invariants are essentially as badly or as well behaved as the group $G$ itself, can be constructed in this way.

The second author introduced recently a concept of property $\mathrm{T}$ for the standard invariant of a subfactor and used certain locally trivial, diagonal subfactors associated to a $G$-kernel to construct reducible, infinite depth subfactors with finite Jones index whose standard invariant has property $\mathrm{T}$ ([Po5], [Po6]). We provide in this note new examples of property $\mathrm{T}$ subfactors by showing that the group type

1991 Mathematics Subject Classification. 46L10, 46L37.

${ }^{1}$ supported by NSF grant DMS-9531566, ${ }^{2}$ supported by NSF grant DMS-9500882 
inclusions $M^{H} \subset M \rtimes K$ have property $\mathrm{T}$ in the sense of [Po6] if and only if the group $G$ has Kazhdan's property T. Once this result is established, every infinite property $T$ group, which is a quotient of a free product of two finite groups, provides an example of an irreducible, infinite depth subfactor of the hyperfinite $\mathrm{II}_{1}$ factor with finite Jones index whose standard invariant has property $\mathrm{T}$. The lowest possible index of an irreducible, infinite depth subfactor with property $\mathrm{T}$ standard invariant that this construction can give is index 6 and we give an example of such a subfactor in section 2, based on work by Conder.

Here is a more detailed description of the two sections below. In section 1 we discuss briefly the different notions of property $\mathrm{T}$ used in this paper and recall a few facts that are needed in the subsequent section. In section 2 we prove that the the standard invariant of the group type subfactors $M^{H} \subset M \rtimes K$ has property T if and only if the group $G$, generated by $H$ and $K$ in Out $M$ has Kazhdan's property $\mathrm{T}$. This is done without explicitly computing the symmetric enveloping inclusion associated to $M^{H} \subset M \rtimes K$. The key fact here is that property $\mathrm{T}$ has certain hereditary properties, which are established in theorem 2.6. The result regarding $M^{H} \subset M \rtimes K$ as mentioned above follows then from these hereditary properties and the fact that a certain locally trivial subfactor associated to the kernel $G$ has property $\mathrm{T}$ in the sense of [Po6] if and only if the group $G$ has Kazhdan's property T. We finish section 2 with a number of explicit examples of subfactors with property $\mathrm{T}$ standard lattice (see corollary 2.9 and remarks).

\section{Property T}

We present in this section three related concepts of property $\mathrm{T}$ used in this paper and fix the notation. Recall first that a countable discrete group $G$ has Kazhdan's property $T[\mathrm{~K}]$ if, roughly speaking, every unitary representation $\pi: G \rightarrow \mathcal{U}(\mathcal{H})$ on a Hilbert space $\mathcal{H}$ that has almost invariant vectors has nonzero invariant vectors. More precisely, $G$ has property T, if there is a finite set $F \subset G$ and there is an $\epsilon>0$ such that whenever $\pi: G \rightarrow \mathcal{U}(\mathcal{H})$ is a unitary representation of $G$ on the Hilbert space $\mathcal{H}$ and $\xi \in \mathcal{H}$ is a unit vector with $\|\pi(s) \xi-\xi\|<\epsilon$, for all $s \in F$, then there is a nonzero vector $\eta \in \mathcal{H}$ with $\pi(g) \eta=\eta$, for all $g \in G$ (see for instance [HV] for other equivalent definitions).

Next we explain two notions of property $T$ related to inclusions of von Neumann algebras. Throughout this paper $N \subset M$ will denote an extremal inclusion of $\mathrm{II}_{1}$ factors with finite Jones index unless otherwise stated. As usual, we denote by $e_{N}$ the orthogonal projection $L^{2}(M) \rightarrow L^{2}(N)$ and by $J: L^{2}(M) \rightarrow L^{2}(M)$ the modular conjugation. The standard invariant of $N \subset M$, consisting of the system of higher relative commutants associated to $N \subset M$, will be denoted by $\mathcal{G}_{N, M}$ (see for instance [Po2]). We refer to $\mathcal{G}_{N, M}$ also as the standard lattice associated to $N \subset M$ (see [Po4]). Inclusions whose index is not necessarily finite will usually be denoted by $\mathcal{N} \subset \mathcal{M}$.

Definition 1.1. ([A-D], [Po1]) Let $\mathcal{N} \subset \mathcal{M}$ be an inclusion of von Neumann algebras and let $\mathcal{M}$ be a $\mathrm{II}_{1}$ factor. We say that $\mathcal{M}$ has property $T$ relative to $\mathcal{N}$, or that the pair $\mathcal{N} \subset \mathcal{M}$ has property $T$, if there is an $\epsilon>0$ and operators $x_{1}, x_{2}, \ldots$, $x_{n} \in \mathcal{M}$ such that if $\mathcal{H}$ is an $\mathcal{M}-\mathcal{M}$ bimodule and $\xi \in \mathcal{H}$ is a vector satisfying 
vector $\eta \in \mathcal{H}$ such that $[\eta, x]=0$ for all $x \in \mathcal{M}$. $\eta$ is called an $\mathcal{M}$-central vector. We call $\left\{\epsilon, x_{1}, \ldots, x_{n}\right\}$ a critical set for $\mathcal{N} \subset \mathcal{M}$.

Note that we do not assume that $\mathcal{N} \subset \mathcal{M}$ has finite Jones index [Jo]. In fact, if $N \subset M$ is an inclusion of $\mathrm{II}_{1}$ factors with finite index, then, using an orthonormal basis of $M$ over $N$ [PiPo], one sees easily that $M$ has property $\mathrm{T}$ relative to $N$. Let us also mention that if $G$ is a countable discrete group with a properly outer action on the $\mathrm{II}_{1}$ factor $N$, then the crossed product $N \rtimes G$ has property T relative to $N$ if and only if the group $G$ has Kazhdan's property T ([A-D], [Po1]).

We recall next the notion of property $\mathrm{T}$ for the standard invariant of an extremal subfactor $N \subset M$ as introduced in [Po5], [Po6]. If $N \subset M$ is an inclusion of $\mathrm{II}_{1}$ factors with finite Jones index, we associate to it the symmetric envelop-

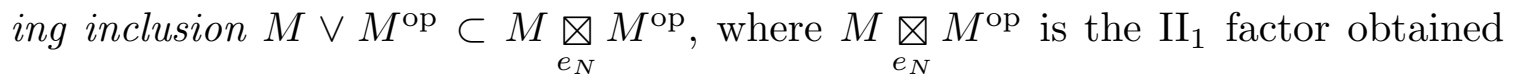
in the following way (see [Po3], [Po6] for details): We consider the $\mathrm{C}^{*}$-algebra $C^{*}\left(M, e_{N}, J M J\right) \subset B\left(L^{2}(M)\right)$, generated by $M, J M J$ and $e_{N}$ on $L^{2}(M)$. It can be shown that $C^{*}\left(M, e_{N}, J M J\right)$ has a unique trace tr and one defines $M \underset{e_{N}}{\otimes} M^{\text {op }}$ to be the von Neumann algebra obtained via the GNS construction with respect to tr from $C^{*}\left(M, e_{N}, J M J\right)$. We can now give the desired definition:

Definition 1.2. [Po6] Let $N \subset M$ be an extremal inclusion of $\mathrm{II}_{1}$ factors with finite Jones index and with standard lattice (or standard invariant) $\mathcal{G}_{N, M}$. We say that $\mathcal{G}_{N, M}$ has property $T$ if the symmetric enveloping $\mathrm{II}_{1}$ factor $M \underset{e_{N}}{\otimes} M^{\text {op }}$ has property $\mathrm{T}$ relative to the subfactor $M \vee M^{\mathrm{op}}$ (in the sense of definition 1.1). Equivalently, we will say that the standard invariant (or the standard lattice) of $N \subset M$ has property $T$.

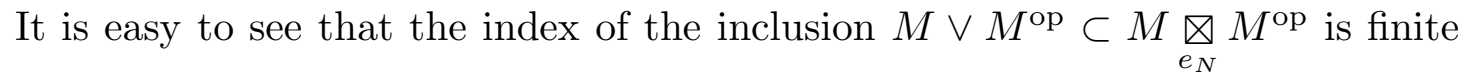
if and only if $N \subset M$ has finite depth. Thus the standard lattice of finite depth subfactors has property $\mathrm{T}$ as expected.

Observe that the definition of property $\mathrm{T}$ for $\mathcal{G}_{N, M}$ depends a priori on the subfactor $N \subset M$. We have however the following result [Po6, section 9]:

Theorem 1.3. Suppose $\mathcal{G}_{N, M}$ is the standard lattice of an extremal subfactor. The property $T$ of $\mathcal{G}_{N, M}$ depends only on the (trace preserving) isomorphism class of $\mathcal{G}_{N, M}$, not on the extremal subfactor from which it was constructed.

If we let $P$ be a $\mathrm{II}_{1}$ factor and $\theta_{0}=i d, \theta_{1}, \ldots, \theta_{n}$ automorphism of $P$, then we can consider the locally trivial inclusion of $\mathrm{II}_{1}$ factors $N^{\theta} \subset M^{\theta}$, where $N^{\theta}=$ $\left\{\sum_{i=0}^{n} \theta_{i}(x) e_{i i} \mid x \in P\right\}, M^{\theta}=P \otimes M_{n+1}(\mathbb{C}),\left\{e_{i j}\right\}_{0 \leq i, j \leq n}$, the matrix units in $M_{n+1}(\mathbb{C})$. These reducible inclusions of $\mathrm{II}_{1}$ factors provide examples of subfactors with property $\mathrm{T}$ standard invariant [Po6, proposition 9.7]:

Proposition 1.4. The standard lattice of the subfactor $N^{\theta} \subset M^{\theta}$ has property $T$ if and only if the group $G$ generated by the $\theta_{i}$ 's, $0 \leq i \leq n$, in the outer automorphism group of $P$ has Kazhdan's property $T$.

The above proposition is proved by computing the symmetric enveloping inclusion associated to $N^{\theta} \subset M^{\theta}$ explicitly. It turns out that it is a crossed product by the group $G$. 


\section{Group type inclusions With Property T}

We study in this section the group type inclusions $M^{H} \subset M \rtimes K$, where $H$ and $K$ are finite groups with a properly outer action on the $\mathrm{II}_{1}$ factor $M[\mathrm{BH}] . M^{H}$ denotes as usual the fixed point algebra under the $H$-action and $M \rtimes K$ the crossed product. Note that $M^{H} \subset M \rtimes K$ has Jones index $|H| \cdot|K|$ and is extremal. It was shown in $[\mathrm{BH}]$ that various analytical properties of these subfactors, such as finite depth, amenability and strong amenability (in the sense of [Po2]), are determined by the group $G=\langle H, K\rangle$, generated by $H$ and $K$ in the outer automorphism group of $M$. Furthermore, $M^{H} \subset M \rtimes K$ is irreducible if and only if $H \cap K=\{e\}$ in Out $M=$ Aut $M / \operatorname{Int} M$. Recall that if $M$ is the hyperfinite $\mathrm{II}_{1}$ factor $R$, then any group $G$, which is a quotient of the free product of $H$ and $K$ gives rise to an irreducible subfactor of the above type. We will show in this section the following theorem:

Theorem 2.1. Let $H$ and $K$ be finite groups with properly outer actions $\sigma$, resp. $\rho$ on the $I I_{1}$ factor $M$. The standard invariant of the subfactor $M^{H} \subset M \rtimes K$ has property $T$ if and only if the group $G$ generated by $\sigma(H)$ and $\rho(K)$ in the outer autmorphism group of $M$ has Kazhdan's property $T$.

This theorem will follow from a more general result, theorem 2.6 below. We will also present at the end of this section some explicit examples of irreducible, infinite depth subfactors whose standard lattice has property $\mathrm{T}$.

The next result can be found in [Po1]. We include the proof here for the convenience of the reader:

\section{Proposition 2.2.}

i) Let $\mathcal{Q} \subset \mathcal{N} \subset \mathcal{M}$ be an inclusion of $I I_{1}$ factors with $[\mathcal{N}: \mathcal{Q}]<\infty$. Then $\mathcal{M}$ has property $T$ relative to $\mathcal{Q}$ if and only if $\mathcal{M}$ has property $T$ relative to $\mathcal{N}$.

ii) Let $\mathcal{N} \subset \mathcal{M}$ be an inclusion of $I I_{1}$ factors and let $p \in \mathcal{N}$ be a nonzero projection. Then $\mathcal{M}$ has property $T$ relative to $\mathcal{N}$ if and only if $p \mathcal{M} p$ has property $T$ relative to $p \mathcal{N} p$.

Proof. Let us first prove i). If $\mathcal{M}$ has property $\mathrm{T}$ relative to $\mathcal{Q}$, then it is trivial that $\mathcal{M}$ has property $\mathrm{T}$ relative to $\mathcal{N}$ - the same critical set works. Conversely, suppose $\mathcal{M}$ has property $\mathrm{T}$ relative to $\mathcal{N}$ and let $\left\{n_{i}\right\}_{i \in I}$ be a (finite) orthonormal basis of $\mathcal{N}$ over $\mathcal{Q}$. Let $\left\{\epsilon, x_{1}, \ldots, x_{n}\right\}, x_{i} \in \mathcal{M}, 1 \leq i \leq n$, be a critical set for $\mathcal{N} \subset \mathcal{M}$ (definition 1.1). Suppose $\mathcal{H}$ is an $\mathcal{M}$ - $\mathcal{M}$-bimodule, $\xi \in \mathcal{H},\|\xi\|=1$ with $[\xi, \mathcal{Q}]=0$. Set $\eta=\sum_{i \in I} n_{i} \xi n_{i}^{*} \in \mathcal{H}$. Then we have for all $x \in \mathcal{N}$

$$
\begin{aligned}
x \eta & =\sum_{i} x n_{i} \xi n_{i}^{*}=\sum_{j} \sum_{i} n_{j} E_{\mathcal{Q}}\left(n_{j}^{*} x n_{i}\right) \xi n_{i}^{*}=\sum_{j} \sum_{i} n_{j} \xi E_{\mathcal{Q}}\left(n_{j}^{*} x n_{i}\right) n_{i}^{*} \\
& =\sum_{j} n_{j} \xi\left(\sum_{i} E_{\mathcal{Q}}\left(n_{j}^{*} x n_{i}\right) n_{i}^{*}\right)=\sum_{j} n_{j} \xi n_{j}^{*} x=\eta x .
\end{aligned}
$$

Furthermore $\left\|n_{i} \xi n_{i}^{*}-\xi n_{i} n_{i}^{*}\right\| \leq\left\|n_{i} \xi-\xi n_{i}\right\|\left\|n_{i}^{*}\right\| \leq[\mathcal{N}: \mathcal{Q}]\left\|n_{i} \xi-\xi n_{i}\right\|$, for all $i$. Thus. if we let $\delta=\max \left\|n_{i} \xi-\xi n_{i}\right\|$ then 


$$
\begin{aligned}
\|\eta-[\mathcal{N}: \mathcal{Q}] \xi\| & =\left\|\sum_{i} n_{i} \xi n_{i}^{*}-\sum_{i} \xi n_{i} n_{i}^{*}\right\| \leq \sum_{i}\left\|n_{i} \xi n_{i}^{*}-\xi n_{i} n_{i}^{*}\right\| \\
& \leq[\mathcal{N}: \mathcal{Q}] \sum_{i}\left\|n_{i} \xi-\xi n_{i}\right\| \leq[\mathcal{N}: \mathcal{Q}]|I| \delta
\end{aligned}
$$

Hence $\|\eta\| \geq[\mathcal{N}: \mathcal{Q}](1-|I| \delta)$, so that $\eta \neq 0$, if $0<\delta<\frac{1}{|I|}$. Fix such a $\delta$ and pick an $\epsilon^{\prime}>0$ with $\epsilon^{\prime}<\min \left\{\delta, \frac{\epsilon(1-|I| \delta)}{3|I|}\right\}$. Then $\left\{\epsilon^{\prime}, x_{i}, x_{i} n_{j}, n_{j}^{*} x_{i}, n_{j}\right\}_{1 \leq i \leq n, j \in I}$ is a critical set for $\mathcal{Q} \subset \mathcal{M}$, since we obtain $\left\|x_{i} \eta^{\prime}-\eta^{\prime} x_{i}\right\|<\epsilon, 1 \leq i \leq n$, where $\eta^{\prime}=\frac{\eta}{\|\eta\|}$ and $\left\{\epsilon, x_{1}, \ldots, x_{n}\right\}$ was the critical set for $\mathcal{N} \subset \mathcal{M}$.

We prove next ii). Let us assume first that $p \mathcal{M} p$ has property $\mathrm{T}$ relative to $p \mathcal{N} p$. Let $\left\{\epsilon, y_{1}, \ldots, y_{n}\right\}, y_{i} \in p \mathcal{M} p$, be a critical set. We may assume $\left\|y_{i}\right\| \leq 1$, $1 \leq i \leq n$. Since $\mathcal{M}$ is a factor, we can find isometries $v_{1}, \ldots, v_{m} \in \mathcal{M}$ such that $v_{1} v_{1}^{*} \leq p, v_{i} v_{i}^{*}=p, 2 \leq i \leq m$ and $\sum_{i=1}^{m} v_{i}^{*} v_{i}=1_{\mathcal{M}}$. Let $\delta=\min \left\{\epsilon / 6, \frac{1}{8 m}\right\}$ and set $\left\{\delta, x_{i}\right\}=\left\{y_{j}\right\}_{1 \leq j \leq n} \cup\left\{v_{j}\right\}_{1 \leq j \leq m} \cup\{p\} \subset \mathcal{M}$. We will show that this consitutes a critical set for $\overline{\mathcal{N}} \subset \mathcal{M}$. Let $\overline{\mathcal{H}}$ be an $\mathcal{M}-\mathcal{M}$ bimodule, $\xi \in \mathcal{H},\|\xi\|=1$ with $[\xi, \mathcal{N}]=0$ and $\left\|\left[\xi, x_{i}\right]\right\|<\delta$, for all $i$. Set $\xi_{0}=p \xi p$. Clearly, $\xi_{0}$ is central for $p \mathcal{N} p$. Note that $\left\|y_{j} \xi_{0}-\xi_{0} y_{j}\right\|=\left\|y_{j} p \xi p-p \xi p y_{j}\right\| \leq\left\|y_{j} \xi p-y_{j} \xi\right\|+\left\|y_{j} \xi-\xi y_{j}\right\|+\| \xi y_{j}-$ $p \xi y_{j}\|\leq 2\| \xi p-p \xi\|+\| y_{j} \xi-\xi y_{j} \|<3 \delta<\epsilon$.

Furthermore $\|\xi\|^{2}=\sum_{i=1}^{m}\left\|v_{i}^{*} v_{i} \xi\right\|^{2} \leq \sum_{i}\left(\left\|v_{i}^{*} v_{i} \xi-v_{i}^{*} \xi v_{i}\right\|^{2}+2\left\|v_{i} v_{i}^{*} \xi v_{i}\right\| \| v_{i} \xi-\right.$ $\left.\xi v_{i}\|+\| v_{i}^{*} \xi v_{i} \|^{2}\right)$. Hence $\|\xi\|^{2} \leq \delta(\delta+2) m+\sum_{i}\left\|v_{i}^{*} \xi v_{i}\right\|^{2}$. But $\left\|v_{i}^{*} \xi v_{i}\right\|^{2} \leq\left\|\xi_{0}\right\|^{2}$, for all $i$, so that $\left\|\xi_{0}\right\|^{2} \geq \frac{1}{m}\|\xi\|^{2}-\delta(\delta+2)$, which is $>0$, if $0<\delta<\frac{1}{4 m}$.

Since $\left\|y_{j} \xi_{0}-\xi_{0} y_{j}\right\|<\epsilon, 1 \leq j \leq n$, there is a vector $\eta_{0} \in p \mathcal{H} p, \eta_{0} \neq 0$, with $\left[\eta_{0}, p \mathcal{M} p\right]=0$. Set $\eta=\sum_{i=1}^{m} v_{i}^{*} \eta_{0} v_{i}$, then it is easy to check that $x \eta=\eta x$, for all $x \in \mathcal{M}$. Since $\|\eta\|^{2} \geq(m-1)\left\|\eta_{0}\right\|^{2}$, we have that $\eta \neq 0$. Thus $\mathcal{M}$ has property $\mathrm{T}$ relative to $\mathcal{N}$.

Conversely, suppose that $\mathcal{M}$ has property $\mathrm{T}$ relative to $\mathcal{N}$. Let $\left\{\epsilon, x_{1}, \ldots, x_{n}\right\}$, $x_{i} \in \mathcal{M}, 1 \leq i \leq n$, be a critical set for $\mathcal{N} \subset \mathcal{M}$. Let $\mathcal{H}_{0}$ be an $p \mathcal{M} p-p \mathcal{M} p$ bimodule and let $\xi_{0} \in \mathcal{H}_{0},\left\|\xi_{0}\right\|=1$, with $\left[\xi_{0}, p \mathcal{N} p\right]=0$. As above, we can find partial isometries $v_{1}, \ldots, v_{m} \in \mathcal{M}$ with $v_{i} v_{i}^{*}=p, 2 \leq i \leq m, v_{1} v_{1}^{*} \leq p$ and $\sum_{i=1}^{m} v_{i}^{*} v_{i}=1$. Consider the set $\left\{\delta, v_{r} x_{k} v_{s}^{*}\right\}_{1 \leq k \leq n, 1 \leq r, s \leq m}$ - it will be a critical set for $p \mathcal{N} p \subset p \mathcal{M} p$, if $\delta$ is small enough. Set $\alpha=\operatorname{tr}(p)^{-1}$ and let $\mathcal{H}$ be the $\alpha$-amplification of $\mathcal{H}_{0}$. The vector $\xi=\sum_{i=1}^{m} v_{i}^{*} \xi v_{i} \in \mathcal{H}$ is $\mathcal{N}$-central and $\neq 0$. We leave it as an exercise to check that $\left\|\xi x_{k}-x_{k} \xi\right\|<\epsilon, 1 \leq k \leq n$, if $\delta$ is chosen small enough. Thus, since $\mathcal{M}$ has property $\mathrm{T}$ relative to $\mathcal{N}$, there is an $\mathcal{M}$-central vector $\eta \in \mathcal{H}, \eta \neq 0$. But then $p \eta p \neq 0$ and $p \eta p \in p \mathcal{H} p=\mathcal{H}_{0}$ is the desired $p \mathcal{M} p$-central vector in $\mathcal{H}_{0}$.

Proposition 2.2 implies the following corollary (see [Po6] for a slightly more general statement):

Corollary 2.3. Let $N \subset M$ be an extremal inclusion of $I I_{1}$ factors with finite index and let $N \subset M \stackrel{e_{1}}{\subset} M_{1}$ be the basic construction. Then the standard invariant of $N \subset M$ has property $T$ if and only if the standard invariant of $M \subset M_{1}$ has property $T$.

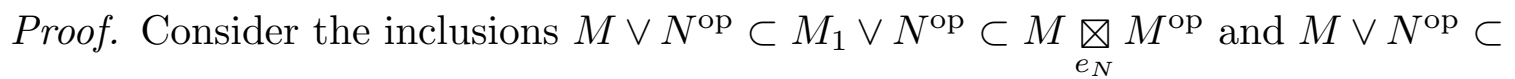
$M \vee M^{\text {op }} \subset M \otimes M^{\text {op }}$. By proposition 2.2 , i) we have that $M \otimes M^{\text {op }}$ has property 


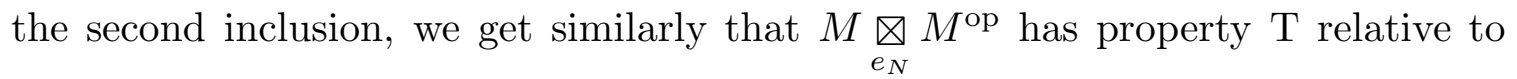

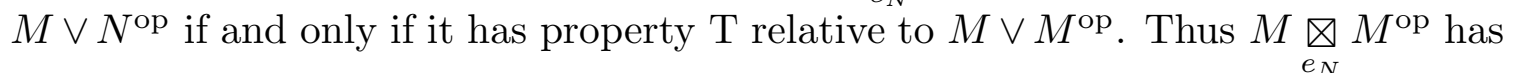
property $\mathrm{T}$ relative to $M \vee M^{\mathrm{op}}$ (i.e. $\mathcal{G}_{N, M}$ has property T) if and only if $M \underset{e_{N}}{\otimes} M^{\mathrm{op}}$ has property $\mathrm{T}$ relative to $M_{1} \vee N^{\mathrm{op}}$. But $M_{1} \vee N^{\mathrm{op}} \subset M \underset{e_{N}}{\otimes} M^{\mathrm{op}}$ is a reduced inclusion of the symmetric enveloping inclusion for $M \subset M_{1}$, reduced by $e_{1}^{\text {op }}$ [Po6, section 2]. By proposition 2.2 ii) we are done.

We also need the following observation from [Po6]:

Lemma 2.4. Let $Q \subset N \subset M$ be an extremal inclusion of $I I_{1}$ factors with finite index and let $\left\{m_{j}\right\}_{j \in J}$ be an orthonormal basis of $N$ over $Q$. The map which sends $e_{N}$ to $\sum_{j} m_{j} e_{Q} m_{j}^{*}$ and which is the identity on $\operatorname{Alg}\left(M, M^{\mathrm{op}}\right)$ implements a unital embedding of $C^{*}\left(M, e_{N}, J M J\right)$ into $C^{*}\left(M, e_{Q}, J M J\right)$ and thus a unital embedding

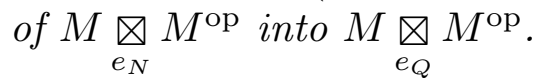

Proof. If $x \in N$, then we have $x=\sum_{j} m_{j} E_{Q}\left(m_{j}^{*} x\right)$ and hence $\sum_{j} m_{j} e_{Q} m_{j}^{*}(\hat{x})$ $=\sum m_{j} E_{Q}\left(m_{j}^{*} x\right)^{\wedge}=\hat{x}$. Also, if $x \in M, \hat{x} \perp N$, then $\widehat{m_{j}^{*} x} \perp N$ as well, so $\sum m_{j} e_{Q} m_{j}^{*}(\hat{x})=\sum_{j} m_{j} e_{Q}\left(\widehat{m_{j}^{*} x}\right)=\sum_{j} m_{j} e_{Q} e_{N}\left(\widehat{m_{j}^{*} x}\right)=0$. Hence $\sum_{j} m_{j} e_{Q} m_{j}^{*}=$ $e_{N}$ in $B\left(L^{2}(M)\right)$. Thus we get $C^{*}\left(M, e_{N}, J M J\right) \subset C^{*}\left(M, e_{Q}, J M J\right)$. By [Po6] it

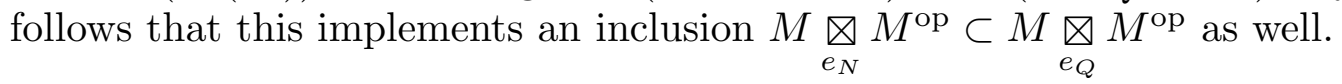

Lemma 2.5. Let $N \subset P \subset M$ be an extremal inclusion of $I I_{1}$ factors with finite index and let $P_{-1} \subset N \subset P \subset M$ be such that $N \subset P$ is the basic construction for $P_{-1} \subset N$. Then $\mathcal{G}_{N, M}$ has property $T$ if and only if $\mathcal{G}_{P_{-1}, M}$ has property $T$.

Proof. By lemma 2.4 we have the inclusion $\left(M \vee M^{\mathrm{op}} \subset M \otimes M^{\mathrm{op}}\right) \subset\left(M \vee M^{\mathrm{op}} \subset\right.$ $\left.M \underset{e_{P}}{\otimes} M^{\mathrm{op}}\right)$. We will show that it is in fact an equality. Clearly, the statement follows then by applying proposition $2.2 \mathrm{i}$ ). Let $N_{1} \subset P_{-1} \subset N \subset P \subset M$ be such that $N \subset M$ is the basic construction for $N_{1} \subset N$ and $N \subset P$ is the basic construction for $P_{-1} \subset N$ (see for instance [Bi]). Then by lemma 2.4 we have the inclusions $C^{*}\left(M, e_{N}, J_{M} M J_{M}\right) \subset C^{*}\left(M, e_{P_{-1}}, J_{M} M J_{M}\right) \subset C^{*}\left(M, e_{N_{1}}, J_{M} M J_{M}\right)$. By [Po6, section 2] the first algebra actually equals the last (using the fact that the Jones projection $M \rightarrow N_{1}$ can be written in terms of the $e_{i}$ 's).

We show next the main theorem of this section.

Theorem 2.6. Let $N \subset P \subset M$ be an extremal inclusion of $I_{1}$ factors with finite index and let $P_{-1} \subset N \subset P \subset M \subset P_{1}$ be such that $N \subset P$ is a basic construction for $P_{-1} \subset N$ and $M \subset P_{1}$ is a basic construction for $P \subset M$. Then the standard invariant of $P_{-1} \subset P_{1}$ has property $T$ if and only if the standard invariant of $N \subset M$ has property $T$. The same statement holds if "property $T$ " is replaced by "amenable".

Proof. Let $P_{-2} \subset N_{1} \subset P_{-1} \subset N \subset P \subset M \subset P_{1}$ be such that $P_{-2} \subset N \subset P_{1}$ and $N_{1} \subset N \subset M$ are basic constructions. Thus the standard invariant of $P_{-2} \subset N$ has property $\mathrm{T}$ if and only if the standard invariant of $N \subset P_{1}$ has property $\mathrm{T}$ (corollary 
property T. By corollary 2.3 we have that $\mathcal{G}_{N, M}$ has property $\mathrm{T}$ if and only if $\mathcal{G}_{N_{1}, N}$ has property $\mathrm{T}$ and the latter has property $\mathrm{T}$ if and only if $\mathcal{G}_{P_{-2}, N}$ does (lemma 2.5 again). Hence, $\mathcal{G}_{N, M}$ has property $\mathrm{T}$ if and only if $\mathcal{G}_{N, P_{1}}$ has property $\mathrm{T}$.

Now consider the inclusions $P_{-1} \subset N \subset P \subset P_{1}$ and apply lemma 2.5 again. Thus $\mathcal{G}_{P_{-1}, P_{1}}$ has property $\mathrm{T}$ if and only if $\mathcal{G}_{N, P_{1}}$ has property $\mathrm{T}$. Together with the above equivalences we have therefore that the standard invariant of $P_{-1} \subset P_{1}$ has property $\mathrm{T}$ if and only if the one of $N \subset M$ has property $\mathrm{T}$.

Using ([Po2], [Po6]) the same proof goes through if "property T" of the standard invariant is replaced by "amenability" of the standard invariant (assuming of course in addition that the $\mathrm{II}_{1}$ factors appearing are hyperfinite, if one replaces "property T" by "amenable inclusions" rather than "amenable standard invariant").

To prove theorem 2.1 we also need the following proposition.

Proposition 2.7. Let $H$ and $K$ be finite groups with properly outer actions $\sigma$, resp. $\rho$ on the $I I_{1}$ factor $P$. Denote $N=P^{H}$ and $M=P \rtimes K$ and let $P_{-1} \subset$ $N \subset P \subset M \subset P_{1}$ be such that $N \subset P$ is the basic construction for $P_{-1} \subset N$ and $M \subset P_{1}$ is the basic construction for $P \subset M$. Then $P_{-1} \subset P_{1}$ is a locally trivial inclusion given by $\left\{\rho_{k} \sigma_{h}\right\}_{h \in H, k \in K}$.

Proof. Note that $P \subset P_{1}$ can be described as follows: $P_{1}=P \otimes B\left(L^{2}(K)\right)$ with matrix units $\left\{e_{k, k^{\prime}}\right\}_{k, k^{\prime} \in K}$ and the inclusion $P \hookrightarrow P_{1}$ is given by $P \ni x \rightarrow$ $\sum_{k \in K} \rho_{k}(x) e_{k, k}$. By perturbing each $\sigma_{h}$ and $\rho_{k}$ by some inner automorphism of $P$ we can assume that they all leave the same $B \subset P$ invariant, with matrix factor $B \cong B\left(l^{2}(H)\right)$ and matrix units $\left\{f_{h, h^{\prime}}\right\}_{h, h^{\prime} \in H}$. But then $P_{-1} \subset P$ is isomorphic to the inclusion $P_{-1} \hookrightarrow P_{-1} \otimes B\left(l^{2}(H)\right)$, given by $P_{-1} \ni x \rightarrow \sum_{h \in H} \sigma_{h}(x) f_{h, h}$. Altogether this gives that $P_{-1} \subset P_{1}$ is isomorphic to the inclusion $P_{-1} \hookrightarrow P_{-1} \otimes$ $B\left(l^{2}(H)\right) \otimes B\left(l^{2}(K)\right)$ given by $P_{-1} \ni x \rightarrow \sum_{h \in H, k \in K} \rho_{k} \sigma_{h}(x) f_{h, h} e_{k, k}$.

Theorem 2.1 follows now immediately from proposition 1.4 and theorem 2.6.

Remark 2.8. Observe that we recovered in theorem 2.6 and proposition 2.7 the result [BH, theorem 4.9]) showing that $P^{H} \subset P \rtimes K$ has amenable principal graph if and only if the group $G$ generated by $\sigma(H)$ and $\rho(K)$ in $\operatorname{Out}(P)$ is amenable.

Numerous explicit examples of infinite groups $G$ that have property $\mathrm{T}$ and are quotients of the free product of two finite groups $H$ and $K$ can be found in [BS] (we would like to thank Pierre de la Harpe for pointing out this reference to us). The construction of these groups is based on Sarnak's examples of Ramanujan graphs [Sa, chapter 3] and an explicit presentation of this class of property $\mathrm{T}$ groups can be found in [BS, theorem 2 and corollary 2]. Since any discrete group has a properly outer action on the hyperfinite $\mathrm{II}_{1}$ factor $R$, we obtain therefore (by applying theorem 2.1) irreducible, infinite depth subfactors of the form $R^{H} \subset R \rtimes K$ whose standard invariant has property $\mathrm{T}$.

We will construct next irreducible, infinite depth subfactors with Jones index 6 whose standard invariant has property T. By theorem 2.1 we need to find an infinite property $\mathrm{T}$ group which is a quotient of $P S L(2, \mathbb{Z})=\mathbb{Z}_{2} * \mathbb{Z}_{3}$.

Corollary 2.9. There are irreducible, infinite depth subfactors of the hyperfinite 
Proof. Let $G$ be the group given by the presentation $\langle x, y| x^{2}=y^{3}=(x y)^{12}=$ $\left.\left(x y^{-1} x y^{-1} x y x y x y^{-1} x y\right)^{2}=1\right\rangle$. It is shown in [Co] that this group is a finite index subgroup of $S L(3, \mathbb{Z}) \rtimes Z_{2}$, where $Z_{2}$ acts on $S L(3, \mathbb{Z})$ as the inverse-transpose automorphism. Since $S L(3, \mathbb{Z})$ has property $\mathrm{T}([\mathrm{K}]$, see also $[\mathrm{HV}])$ and property $\mathrm{T}$ is preserved under finite extensions and finite index subgroups (see for instance $[\mathrm{HV}]$ ), the group $G$ must have property $\mathrm{T}$ as well. $G$ is the desired infinite quotient of $\operatorname{PSL}(2, \mathbb{Z})$ with property $\mathrm{T}$ - apply then theorem 2.1 .

Remark 2.10.

i) In $[\mathrm{CRW}] S L(3, \mathbb{Z})$ is written as a quotient of $A_{4} * \mathbb{Z}_{3}$. By theorem 2.1 we obtain therefore an irreducible, infinite depth subfactor with index 36 and property $\mathrm{T}$ standard invariant.

ii) In $[\mathrm{CRW}] S L(3, \mathbb{Z}) \rtimes \mathbb{Z}_{2}$ is written as a quotient of $\left(\mathbb{Z}_{2} \times \mathbb{Z}_{2}\right) * \mathbb{Z}_{3}$. Again by theorem 2.1, we obtain an irreducible, infinite depth subfactor with index 12 and property $\mathrm{T}$ standard invariant and intermediate subfactors of index 2 and 6.

We end this paper by mentioning a few problems that are closely related to the above work and seem to be of interest at this point.

a) Are there irreducible, infinite depth subfactors with property $\mathrm{T}$ standard lattice and Jones index $<6$ ? Observe that such a subfactor would have necessarily index $>3+\sqrt{3}$ by [Ha] and [Po6, section 9].

b) The group type inclusions $M^{H} \subset M \rtimes K$ have intermediate subfactors. Construct irreducible, infinite depth subfactors whose standard lattice has property $\mathrm{T}$ that do not have intermediate subfactors.

c) Construct irreducible, infinite depth subfactors with noninteger Jones index whose standard lattice has property $\mathrm{T}$.

d) It would be interesting to compute the symmetric enveloping inclusion associated to $M^{H} \subset M \rtimes K$ and to give then a different proof of our theorem 2.1 .

e) Suppose $N \subset P \subset M$ is an extremal inclusion of $\mathrm{II}_{1}$ factors with finite index such that the standard lattice of $N \subset M$ has property T. Suppose furthermore that one of the intermediate inclusions has finite depth. Does it then follow that the standard lattice of the other intermediate inclusion has property $\mathrm{T}$ ? The answer may very well be negative. 


\section{REFERENCES}

[A-D] C. Anantharaman-Delaroche, On Connes' property T for von Neumann algebras, Math. Japonica 3 (1987), 337-355.

[BS] W. Ballman, J. Swiatkowski, On $L^{2}$-cohomology and property (T) for automorphism groups of polyhedral cell complexes, Geom. funct. anal. 7 (1997), 615-645.

[Bi] D. Bisch, A note on intermediate subfactors, Pacific Journal of Math. 163 (1994), 201-216.

[BH] D. Bisch, U. Haagerup, Composition of subfactors: new examples of infinite depth subfactors, Ann. scient. Éc. Norm. Sup. 29 (1996), 329-383.

[Co] M. Conder, A surprising isomorphism, Journal of Algebra 129 (1990), 494-501.

[CRW] M. Conder, E. Robertson, P. Williams, Presentations for 3-dimensional special linear groups over integer rings, Proceedings of the American Math. Soc. 115 (1992), 19-26.

[Ha] U. Haagerup, Principal graphs of subfactors in the index range $4<[M: N]<3+\sqrt{2}$, in Subfactors (Kyuzeso, 1993), World Sci. Publishing, 1994, pp. 1-38.

[HV] P. de la Harpe, A. Valette, La propriété (T) de Kazhdan pour les groupes localement compacts, Astérisque 175 (1989).

[Jo] V.F.R. Jones, Index for subfactors, Invent. Math. 72 (1983), 1-25.

[K] D. Kazhdan, Connections of the dual space of a group with the structure of its closed subgroups, Funct. Anal. and its Appl. 1 (1967), 63-65.

[PiPo] M. Pimsner, S. Popa, Entropy and index for subfactors, Ann. scient. Ec. Norm. Sup. 19 (1986), 57-106.

[Po1] S. Popa, Correspondences, INCREST preprint (1986).

[Po2] S. Popa, Classification of amenable subfactors of type II, Acta Math. 172 (1994), 352-445.

[Po3] S. Popa, Symmetric enveloping algebras, amenability and AFD properties for subfactors, Mathematical Research Letters 1 (1994), 409-425.

[Po4] S. Popa, An axiomatizaton of the lattice of higher relative commutants, Invent. Math. 120 (1995), 427-445.

[Po5] S. Popa, Amenability in the theory of subfactors, in "Operator Algebras and Quantum Field Theory", International Press, editors S. Doplicher et al., 1997, pp. 199-211.

[Po6] S. Popa, Some properties of the symmetric enveloping algebra of a subfactor, with applications to amenability and property T, preprint 1997.

[Sa] P. Sarnak, Some applications of modular forms, Cambridge Tracts in Mathematics, vol. 99, Cambridge University Press, 1990.

UC Santa Barbara, Department of Mathematics, Santa Barbara, CA 93106, USA and Université de Genève, Section de mathématiques, 2-4, Rue du Lievre, Case postale 124, CH-1211 Genève 24, Switzerland

E-mail address: bisch@math.ucsb.edu, Sorin.Popa@math.unige.ch, popa@math.ucla.edu 